\title{
New American and European Hypertension Guidelines, Reconciling the Differences
}

\author{
Alejandro de la Sierra
}

Received: June 21, 2019 / Published online: July 30, 2019

(c) The Author(s) 2019 important changes in therapeutic aspects, including treatment initiation and blood pressure goals for treated patients. In this review, the main differences between American and European recommendations are highlighted, along with the arguments exposed by both groups of experts and their possible impact affecting clinical practice in hypertension management.

Keywords: Antihypertensive treatment; Blood pressure categories; Cardiovascular risk; Hypertension; Hypertension definition

\section{INTRODUCTION}

Among all the risk factors involved in mortality and disability worldwide, hypertension is the one with the greatest impact, regarding its responsibility in the development of cardiovascular disease, which represents the main cause of death in all developed and many developing countries [1].

The scientific evidence supporting the benefits of pharmacological treatment of hypertension has been developing for more than 60 years. Many hypertensive patients have participated in clinical trials, most of them with a high quality of design, showing the benefits of such treatments in the prevention of both the onset and progression of cardiovascular disease,

\author{
A. de la Sierra $(\square)$ \\ Hypertension Unit, Department of Internal \\ Medicine, Hospital Mútua Terrassa, University of \\ Barcelona, Barcelona, Spain \\ e-mail: adelasierra@mutuaterrassa.cat; \\ asierra@ub.edu
}

Enhanced Digital Features To view enhanced digital \begin{tabular}{l} 
m9.figshare.8938058. \\
\hline
\end{tabular} 
as well as in the prevention of mortality due to cardiovascular causes.

Both official agencies, depending on governmental organizations and scientific societies, have developed recommendations for the diagnosis, evaluation, and treatment of hypertension, resulting in a way of trying to assist physicians who usually take care of hypertensive patients, either in primary care or in referral units. Among the different clinical guidelines produced in several parts of the world (at local, national, or international levels), there is no doubt that the initiatives developed either in the United States or in Europe are those raising a greater expectation and a wider diffusion.

The history of clinical guidelines in the US begins with the Joint National Committee (JNC), an organization created in 1972 by the US government through the National Institutes of Health (NIH). The first report, known as JNC 1 , was published in 1977 , and since then the recommendations have been updated periodically until the year 2003, when the seventh report was released [2]. Although a group of panelists were working on a new report, in mid2013, the NIH decided to transfer the responsibility of creating new guidelines to the corresponding scientific societies. In the case of hypertension, these were the American College of Cardiology (ACC) and the American Heart Association (AHA). The result was the publication, at the end of 2017, of an extensive clinical guide [3]. European guidelines were first released in 2003 by the creation of a joint committee from the European Society of Hypertension and the European Society of Cardiology. New updated versions were published in 2007, 2013, and more recently in 2018 [4].

These two recent published guidelines from US and Europe have created some controversy, as they contain different recommendations from the same clinical situations, after reviewing what apparently is the same evidence. It should be recognized that both guidelines contain more similarities than differences, and that some of these differences can be attributed to local epidemiological aspects or related to particularities about the type of health care provided on both sides of the Atlantic Ocean.
However, differences that affect some fundamental aspects also appear. These include the classification of blood pressure and diagnosis of hypertension, the evaluation of cardiovascular risk, the usefulness of out-of-office blood pressure (BP) measurements, as well as therapeutic aspects such as treatment initiation, blood pressure goals, first-line drugs, or the use of combination therapies as the initial treatment. In this review, we will briefly discuss some of these differential aspects between the two guidelines. This article is based on previously conducted studies and does not contain any studies with human participants or animals performed by any of the authors.

\section{Blood pressure categories and definition of hypertension}

Previous versions of both American [2] and European [5] guidelines defined hypertension with $\mathrm{BP}$ values $\geq 140 / 90 \mathrm{mmHg}$. The JNC VII introduced the term pre-hypertension to define subjects with BP between 120-139 and/or 80-$89 \mathrm{mmHg}^{2}$. The term was based on the concept that the residual risk for developing hypertension among these subjects was very high, approaching 85-90\% [6].

In its current version [4], the European guidelines maintain the same classification of BP categories contained in previous documents ${ }^{5}$. Hypertension continues to be defined as $\mathrm{BP}$ greater than $140 / 90 \mathrm{mmHg}$, with hypertensive patients divided into three categories (grades 1,2, or 3), depending on the magnitude of BP elevation. Normotensives are also separated as optimal $(<120 / 80 \mathrm{mmHg})$, normal (120-129/80-85 mmHg), or high-normal (130-139/85-89 mmHg) categories. According to the European experts, the review of the literature does not justify any modification.

The American guidelines [3] substantially modified the threshold values for hypertension definition to $\geq 130 / 80 \mathrm{mmHg}$, which represents the most important change in the last 20 years. The categories within the hypertensive group are reduced to two (stage 1, defined as BP values between 130-139 and/or $80-89 \mathrm{mmHg}$ and stage $2, \geq 140 / 90 \mathrm{mmHg}$ ). Within the non- 
Table 1 Blood pressure classification in the ACC/AHA and ESC/ESH guidelines

\begin{tabular}{|c|c|c|c|c|c|c|c|}
\hline \multicolumn{4}{|l|}{ ACC/AHA } & \multicolumn{4}{|l|}{ ESC/ESH } \\
\hline Category & SBP & & DBP & Category & SBP & & DBP \\
\hline Normal & $<120$ & and & $<80$ & Optimal & $<120$ & and & $<80$ \\
\hline Elevated & $120-129$ & and & $<80$ & Normal & $120-129$ & and/or & $80-84$ \\
\hline Stage 1 hypertension & $130-139$ & or & $80-89$ & High-normal & $130-139$ & and/or & $85-89$ \\
\hline \multirow[t]{4}{*}{ Stage 2 hypertension } & $\geq 140$ & or & $\geq 90$ & Grade 1 hypertension & $140-159$ & and/or & $90-99$ \\
\hline & & & & Grade 2 hypertension & $160-179$ & and/or & $100-109$ \\
\hline & & & & Grade 3 hypertension & $\geq 180$ & and/or & $\geq 110$ \\
\hline & & & & Isolated systolic hypertension & $\geq 140$ & and & $<90$ \\
\hline
\end{tabular}

hypertensive categories, subjects with systolic BP (SBP) between 120 and 129, and diastolic BP (DBP) $<80 \mathrm{mmHg}$, are classified as having elevated BP. Normal BP is considered when values are $<120 / 80 \mathrm{mmHg}$ (Table 1 contains both classifications).

The American experts justify this reduction in the threshold value for hypertension definition mainly on epidemiological reasons. First, hypertension continues to be the main risk factor producing death and disability. Secondly, several meta-analyses indicate that the risk of coronary events and stroke among subjects with SBP between 130 and 139 is $1.5-2$ times higher compared to those who have SBP below 120 . Moreover, although this increased risk attenuates with advanced age, it is still present in older adults [7]. According to this data, the American experts consider an urgent need to establish preventive measures in these groups, both to reduce the impact of cardiovascular disease and to avoid future progression towards more advanced stages of BP elevation. In contrast, the European point of view is more conservative. Data on antihypertensive treatment in individuals with BP levels below 140/90 $\mathrm{mmHg}$ has not shown a questionable benefit. In regard to this, the European experts do not consider defining an individual as belonging to an "abnormal" category (hypertension) if there is not a clear indication for treatment.

The epidemiological impact of this change in the definition of hypertension stated in the American guidelines has been quantified in the
US, where the prevalence of hypertension would increase from 32 to $46 \%$ [8].

\section{Evaluation of cardiovascular risk in the hypertensive patient}

Several factors are recognized to influence the total cardiovascular risk in hypertensive patients. Some of them, such as smoking, diabetes, or dyslipidemia, are more prevalent in hypertensives in comparison to the general population. The presence of such comorbidities has a double implication. To start with, patient management includes treatment of all potentially modifiable risk factors, not only BP elevation. On the other hand, the initiation and intensity of the antihypertensive treatment may be influenced by other risk factors and thus by the total cardiovascular risk.

The sixth report of the JNC [9], which appeared at the end of 1997, mentioned for the first time a risk stratification for hypertension. Patients were classified into three categories: A, $B$ and $C$, based on the estimation of their absolute risk. Each with different therapeutic recommendations, depending on which category an individual was placed in. This concept was also adopted by the European guidelines, first appearing in 2003 and maintained until the last version.

Both the ACC/AHA [3] and the ESC/ESH [4] guidelines consider the evaluation of total cardiovascular risk as an important tool affecting the onset and the intensity of antihypertensive 
treatment. The American guidelines proposes the use of a calculation derived from the equation of a pooled cohort [10], which comprised the Framingham cohort, the ARIC study (Atherosclerosis Risk in Communities), the Cardiovascular Health Study, and the CARDIA study (Coronary Artery Risk Development in Young Adults). This equation calculates the risk of a first fatal or non-fatal cardiovascular event at 10 years based on a series of parameters including BP, lipid levels, the presence of diabetes, age, sex, race, smoking, and treatments received for such conditions. Its main advantage is that the equation is also useful in African Americans and that it predicts the risk of any cardiovascular event, fatal or not.

The European guideline continues to use the equations provided by the SCORE (Systematic Coronary Risk Evaluation) study [11], which calculates the risk of a fatal cardiovascular event. Its main advantage is that it has been developed on the basis of the European population, hence equations are adapted to specific European countries (the risk is heterogeneous between northern and southern European countries). Its greatest disadvantage is that the risk is restricted to fatal episodes. Since it is well known that the fatality of many of these episodes is clearly influenced by the quality of care provided, especially in regard to early detection and management, including revascularization. In the European guidelines, this limitation is mentioned and an approximate calculation of the risk of non-fatal episodes is provided, multiplying the risk value obtained in SCORE by 3 in men, and by 4 in women [4].

In the risk classification proposed by the European guidelines, there are two particularities that were not considered in the American guidelines. First, it is stated that some patients should be considered directly as having very high risk due to the presence of documented cardiovascular disease (even if it is asymptomatic), or to the presence of diabetes, chronic kidney disease, or very high values of a single risk factor (grade 3 hypertension, family hypercholesterolemia, etc.). Secondly, it includes hypertension-mediated silent organ damage (left ventricular hypertrophy, microalbuminuria, arterial stiffness, etc.), as risk modifiers, although these elements are not included in the risk calculation of the SCORE system. Consistent in both guidelines is that the risk calculation will be decisive, together with the degree of elevation of BP in the initiation of antihypertensive treatment.

\section{Out-of-office blood pressure measurement}

The use of home BP monitoring (HBPM) and ambulatory BP monitoring (ABPM) is for the first time recognized in the US guidelines as an important tool for both diagnosis and management of elevated BP. In individuals whose BP at the office is between 130 and 160 and/or between 80 and $100 \mathrm{mmHg}$, the use of HBPM or $\mathrm{ABPM}$ is recommended to confirm such BP elevation or to discard white-coat hypertension [3]. Moreover, in this latter group, the initiation of antihypertensive medication is not recommended. In parallel, in normotensive subjects with elevated BP $(120-129 /<80 \mathrm{mmHg})$, HBPM or ABPM could be indicated to detect masked hypertension, also with a recommendation of antihypertensive treatment initiation in those with out-of-office BP above 130/80 $\mathrm{mmHg}$.

The same recommendation applies to hypertensive patients receiving antihypertensive treatment. In those not controlled with three different antihypertensive drugs, HBPM or ABPM should be performed, as one-third of them could have white-coat-resistant hypertension [12]. Furthermore, in treated and controlled patients, which remain at high cardiovascular risk or with persistent silent organ damage, the possibility of $\mathrm{MUCH}$ (masked uncontrolled hypertension) should be ruled out. Again, almost one-third of such patients may have this condition [13], which carries an important risk of increased mortality [14], thus requiring treatment intensification [3].

The European guidelines [4] continues to mention, as in previous editions, that office measurement remains the standard method for the diagnosis and monitoring of the hypertensive patient. With emphasis on the need for measuring BP after $1 \mathrm{~min}$ and $3 \mathrm{~min}$ of standing position in sensitive populations, such as the 
elderly, diabetics, or other clinical conditions that favor orthostatic hypotension. This condition is defined as a fall in BP $>20 \mathrm{mmHg}$ for SBP or $>10 \mathrm{mmHg}$ for DBP. With respect to HBPM and ABPM, the European guidelines already highlighted the potential value of such measurement methods in previous versions [5]. This is reinforced in the 2018 document [4] following the recommendations of a previous position paper from the ESH [15]. The main recommendations are similar to those of the US guidelines, considering treatment initiation and treatment intensification in patients with masked hypertension and $\mathrm{MUCH}$, respectively. With respect to white-coat hypertension, although recognizing that antihypertensive treatment is not routinely recommended, it could be appropriate for white-coat hypertensives with a high cardiovascular risk, due to the presence of other cardiovascular risk factors or hypertensivemediated organ damage.

The main differences between the two guidelines refer to normal values of out-of-office BP. The European guidelines retain classical values of $135 / 85 \mathrm{mmHg}$ for home and daytime BP, $\quad 130 / 80 \mathrm{mmHg}$ for 24-h BP, and $120 / 70 \mathrm{mmHg}$ for night-time $\mathrm{BP}$, derived from previous observational studies. In contrast, the US guidelines were obliged to reduce the threshold for normality of out-of-office BP, in accordance to the new hypertension definition based on office BP values below 130/80 $\mathrm{mmHg}$. However, these new values do not clearly derive from any previous evidence. Table 2 contains the thresholds of both guidelines.

Table 2 Thresholds for hypertension definition based on clinic, home, and ambulatory blood pressure

\begin{tabular}{lll}
\hline & ACC/AHA & ESC/ESH \\
\hline Clinic & $130 / 80$ & $140 / 90$ \\
Home & $130 / 80$ & $135 / 85$ \\
Daytime & $130 / 80$ & $135 / 85$ \\
$24-h$ & $125 / 75$ & $130 / 80$ \\
Night-time & $110 / 65$ & $120 / 70$ \\
\hline
\end{tabular}

\section{Antihypertensive treatment initiation- impact of blood pressure, age, and total cardiovascular risk}

Both European and American guidelines base the decision concerning treatment initiation on the degree of BP elevation and the absolute cardiovascular risk. The review of evidence from randomized controlled trials shows that the relative risk reduction obtained with treatment is constant in all risk strata. Moreover, absolute risk reduction is proportional to the baseline risk [16].

Both guidelines also agree when considering that pharmacological treatment must always be accompanied by personalized advice on lifestyle changes. This advice should be emphasized at not only the first visit but also each time the patient has contact with a health care provider. Weight loss in overweight individuals, reduction of salt intake, adoption of a healthy diet with increase in the consumption of fruits and vegetables and a parallel reduction in processed foods, promotion of physical exercise, and smoking cessation are all measures that should be implemented in the management of hypertensive patients.

Pharmacological treatment initiation is recommended by both European and American guidelines in hypertensive patients with $\mathrm{BP}$ $\geq 160 / 100 \mathrm{mmHg}$ (HTA grades 2 and 3 of the European classification). According to the American guidelines, all patients with BP $\geq 140 / 90 \mathrm{mmHg}$ (stage 2 hypertension) should receive antihypertensive treatment, regardless of age or baseline cardiovascular risk. The only exception is the presence of normal HBPM or ABPM (white-coat hypertension). In the European guidelines, treatment of patients with grade 1 hypertension (140-159/90-99 $\mathrm{mmHg}$ ) is influenced by age and the total baseline cardiovascular risk. The recommendation in patients with high or very high cardiovascular risk (with cardiovascular disease, chronic kidney disease or target organ damage) is, like in the American guidelines, to initiate pharmacological treatment without delay. In patients with lower risk, antihypertensive treatment is considered reasonable if BP remains above 
$140 / 90 \mathrm{mmHg}$ after 3-6 months of lifestyle changes.

In addition, in patients older than 65 years, pharmacological treatment is recommended only with systolic $\mathrm{BP} \geq 160$, although such a possibility is considered for those with systolic BP between 140 and $160 \mathrm{mmHg}$ aged 65-80 years, provided that the patient is in acceptable general health conditions and the treatment is adequately tolerated.

Differences in the definition of hypertension also extrapolate to distinct recommendations in individuals with systolic $\mathrm{BP}<140 \mathrm{mmHg}$. As already mentioned, the group with BP 130-139/ $80-89 \mathrm{mmHg}$ is classified as having stage 1 hypertension in the US and pharmacological therapy is recommended for those with established cardiovascular disease or with an absolute cardiovascular risk of more than $10 \%$ at 10 years, by using the pooled cohort equation. The remaining individuals are advised to adopt lifestyle changes and to be periodically monitored for the progression to stage 2 hypertension.

These individuals, classified as having highnormal BP in the European guidelines (categories are not exactly the same as the range of DBP in Europe is $85-89 \mathrm{mmHg}$ ), are equally advised to adopt healthy lifestyles and, in general, antihypertensive treatment is not recommended, with the exception of those with documented coronary heart disease. Table 3 summarizes the recommendations of both guidelines with respect to treatment initiation.

It is relatively surprising that both guidelines refer to the same randomized controlled trials to produce different recommendations. There are two aspects which deserve attention. On the one hand, the greater aggressiveness of the American guidelines, aligned with changes in hypertension definition, is intended to reduce the burden of hypertensive disease and the consequences of cardiovascular disease, thus prevailing the epidemiological perspective. In the current context of a generally safe, welltolerated, and affordable antihypertensive medication, it is reasonable to expect more advantages than disadvantages in taking such a decision. There is no doubt that a general reduction of BP in the general population,
Table 3 Antihypertensive treatment initiation

\begin{tabular}{|c|c|c|}
\hline & ACC/AHA & ESC/ESH \\
\hline $\begin{array}{l}\mathrm{BP} \geq 160 \\
\text { and } / \\
\text { or } \geq 100\end{array}$ & $\begin{array}{l}\text { Lifestyle changes } \\
\text { and } \\
\text { antihypertensive } \\
\text { treatment }\end{array}$ & $\begin{array}{l}\text { Lifestyle changes } \\
\text { and } \\
\text { antihypertensive } \\
\text { treatment }\end{array}$ \\
\hline $\begin{array}{l}\text { BP } 140-159 \\
\text { and/or } \\
90-99 \text { with } \\
\text { high or very } \\
\text { high risk }\end{array}$ & $\begin{array}{l}\text { Lifestyle changes } \\
\text { and } \\
\text { antihypertensive } \\
\text { treatment }\end{array}$ & $\begin{array}{l}\text { Lifestyle changes } \\
\text { and } \\
\text { antihypertensive } \\
\text { treatment }\end{array}$ \\
\hline $\begin{array}{l}\text { BP 140-159 } \\
\text { and/or } \\
\text { 90-99 with } \\
\text { low risk }\end{array}$ & $\begin{array}{l}\text { Lifestyle changes } \\
\text { and } \\
\text { antihypertensive } \\
\text { treatment }\end{array}$ & $\begin{array}{l}\text { Lifestyle changes } \\
\text { Consider } \\
\text { antihypertensive } \\
\text { treatment if BP } \\
\text { remains elevated } \\
\text { after 3-6 months }\end{array}$ \\
\hline $\begin{array}{l}\text { BP } 130-139 \\
\text { and/or } \\
80-89^{\mathrm{a}}\end{array}$ & $\begin{array}{l}\text { Lifestyle changes } \\
\text { and } \\
\text { antihypertensive } \\
\text { treatment if } \\
\text { CVD or elevated } \\
\text { CV risk ( }>10 \% \\
\text { in } 10 \text { years) }\end{array}$ & $\begin{array}{l}\text { Lifestyle changes } \\
\text { Consider } \\
\text { antihypertensive } \\
\text { treatment in very } \\
\text { high risk patients } \\
\text { due to coronary } \\
\text { heart disease }\end{array}$ \\
\hline
\end{tabular}

CVD cardiovascular disease

a In the European guidelines, this recommendation applies to patients with SBP 130-139 and/or DBP 85-89

including hypertensive patients, will translate into a reduction of cardiovascular deaths and disability.

The European perspective is more focused on the individual patient than in the global population. The most conservative attitude tries to avoid treatment in individuals where the benefit has not been unequivocally proven. In fact, the most important recent evidence in hypertension, the SPRINT (Systolic Blood Pressure Intervention Trial) study [17], does not have a uniform impact in both guidelines. The SPRINT trial, promoted by the NIH and developed entirely in the US, included patients older than 50 years with systolic $\mathrm{BP} \geq 130 \mathrm{mmHg}$ (more 
than half had values between 130 and $140 \mathrm{mmHg}$ ). Results demonstrated that the group randomized to a systolic BP $<120 \mathrm{mmHg}$ significantly reduced the occurrence of both total mortality and cardiovascular events. Obviously, the trial strongly influenced the new American guidelines, while from the European perspective it has received more criticisms. Among them, it has been argued that most patients included were previously under antihypertensive treatment and so this systolic BP between 130 and $140 \mathrm{mmHg}$ cannot be considered as their baseline BP. According to this argument, the results of the trial could not be extended to the untreated population at the time of treatment initiation.

\section{Therapeutic objectives of antihypertensive treatment}

For the first time, the ACC/AHA guidelines [3] recommended values below $130 / 80 \mathrm{mmHg}$ as a therapeutic target for all hypertensive patients. This recommendation is classified as class I and therefore mandatory, for patients with a high cardiovascular risk (established cardiovascular disease or a predicted risk of cardiovascular events higher than $10 \%$ in 10 years) and reasonable (class IIb recommendation) for those with lower risk.

In adopting this strategy, more aggressive than that of previous guidelines, the results of the aforementioned SPRINT trial [17], demonstrating a clear benefit in patients randomized to a lower BP target, have been of great influence. Differences in BP between the therapeutic target of the intensive treatment group of the SPRINT trial $(<120 \mathrm{mmHg})$ and that proposed by the guidelines $(<130 \mathrm{mmHg})$ is justified by the unattended BP measurement method used in SPRINT. It has been suggested that this measurement method results in lower BP values in comparison to standard attended measurements [18]. From a practical point of view, values of $120 \mathrm{mmHg}$ obtained with the unattended measurement would be close to 130 in clinical practice.

It could be criticized that the generalization of all the hypertensive population of such BP treatment target does not correspond with the SPRINT data, where only patients older than 50 years with additional cardiovascular risk factors were included. Besides, both diabetics and patients with a previous stroke were excluded from the SPRINT. Two previous studies $[19,20]$ with the same treatment design (comparison of an intensive versus a conventional BP target) in diabetics and patients with a previous lacunar stroke did not result in a clear benefit, with the exception of some secondary endpoints (prevention of stroke in diabetics and prevention of hemorrhagic cerebrovascular events in patients with a previous stroke). In this context, and also aligned with the new hypertension definition, the authors of the American guidelines considered that this evidence, along with the results of several recent meta-analyses, was important enough to establish a goal below 130/80 $\mathrm{mmHg}$ for the majority of the hypertensive population.

The European guidelines are less strict regarding therapeutic goals but also more detailed, with different targets depending highly on age. In fact, the general recommendation is structured into two sentences. It is stated that the first therapeutic objective should be to reduce BP to values below 140/90 $\mathrm{mmHg}$, but an immediately followed second objective of values below $130 / 80 \mathrm{mmHg}$ is mentioned, providing that antihypertensive treatment is well tolerated. The European guidelines also emphasize that a reduction of SBP below $120 \mathrm{mmHg}$ should be avoided and in patients older than 65 , a reasonable target is to maintain SBP between 130 and $140 \mathrm{mmHg}$.

The main conclusion is that both recommendations, ACC/AHA [3] and ESC/ESH [4], are similar, and are only different in how they are formulated. The American perspective is more pragmatic, containing clear and concise recommendations for most hypertensive patients. The European guidelines are more cautious. They try to achieve the same objectives, but avoid a possible negative impact of excessive BP reduction, especially in older and frail patients. The consequences of either one of the strategies in hypertension control and disease-related complications should be analyzed in the future. 
First-line antihypertensive drug classes and agents

The selection of first-line therapeutic agents has usually been divergent between American and European guidelines. While the European guidelines included several antihypertensive drug classes (diuretics, beta-blockers, calcium channel blockers, angiotensin-converting enzyme (ACE) inhibitors, and angiotensin-receptor blockers) as suitable for treatment initiation [5], previous reports from the JNC [2] advocated for a preferential use of diuretics, alone or in combination. In their current version, both guidelines converged to this wide approach considering several drug classes as first-line agents. The only discrepancy refers to beta-blockers, which are still considered firstline agents in the European guidelines, but restricted to specific indications, such as angina, post myocardial infarction, congestive heart failure, supraventricular arrhythmias requiring heart rate control, or young women who are planning pregnancy.

Another additional difference relates to the selection of the diuretic agent. While both guidelines advocate for the preferential use of thiazide and thiazide-like diuretics, with respect to loop diuretics, only the ACC/AHA guidelines recommend a preferential use of clorthalidone versus hydrochlorothiazide. Chlorthalidone has been the thiazide-like diuretic used in the ALLHAT (Antihypertensive and Lipid-Lowering Treatment to Prevent Heart Attack Trial) study [21], resulting in similar benefits when compared to the ACE inhibitor lisinopril, or the calcium channel blocker amlodipine. Having said that, a recent meta-analysis comparing both drugs did not result in any significant advantage of chlorthalidone [22].

\section{Monotherapy or combination therapy for treatment initiation}

The classical approach to antihypertensive treatment recommended a single agent for treatment initiation, and the addition of other agents if BP control was not achieved. This stepped-care strategy was questioned in the previous JNC VII [2], where two-drug combination was proposed for treatment initiation in selected patients. Accordingly, previous versions of the European guidelines [5] also introduced the possibility of initial combination therapy in patients with hypertension grades 2 or 3 , or with high cardiovascular risk. In the current version of both American and European guidelines, combination therapy has become the preferred option for treatment initiation for the majority of patients. In the ACC/AHA guidelines [3], this is the recommended option for patients with stage 2 hypertension (values above $140 / 90 \mathrm{mmHg}$ ). In the ESC/ESH guidelines [4], this is the preferred option for most patients, excluding frail, older patients, or those with grade 1 hypertension and low cardiovascular risk.

With regard to the components of such combinations, both guidelines recommend the use of ACE inhibitors or angiotensin-receptor blockers (but never combining both classes), with either diuretics or calcium channel blockers. The European guidelines add some particularities, by considering that these combinations should be, if possible, administered in one single pill, given its beneficial impact of treatment adherence [23]. In addition, the use of a triple therapy on patients not controlled with two-drug combinations is also advocated to be administered in a single pill containing an ACE inhibitor or an angiotensinreceptor blocker; a diuretic and a calcium channel blocker.

\section{CONCLUSIONS}

In summary, the main differences between ACC/AHA and ESC/ESH guidelines derive from the new definition of hypertension stated in the US with values $\geq 130 / 80 \mathrm{mmHg}$, while European guidelines retain the classical threshold of $140 / 90 \mathrm{mmHg}$. This difference in definition carries variant approaches with respect to treatment initiation and therapeutic objectives. US guidelines are clearer, pragmatic, and the main objective is to reduce the burden of hypertension-related disease by trying to detect risk individuals at an earlier stage, all while 
being more interventionist in reducing the risk of such individuals and accordingly, the cardiovascular health of the general population. In contrast, the European perspective is more conservative, less focused on epidemiological issues, but more addressed to the individual patient. In turn, trying to personalize the treatment, as to avoid such treatment for those who will not obtain a clear benefit, or for those who an excessive BP reduction may be harmful to. Two different strategies are confronted. The coming years will help elucidate which one better fits to the reduction of cardiovascular morbidity and mortality.

\section{ACKNOWLEDGEMENTS}

Funding. No funding or sponsorship was received for this study or publication of this article.

Authorship. The named author meets the International Committee of Medical Journal Editors (ICMJE) criteria for authorship for this article, takes responsibility for the integrity of the work as a whole, and has given their approval for this version to be published.

Disclosures. Alejandro de la Sierra has nothing to disclose. Alejandro de la Sierra is a member of the journal's Editorial Board.

Compliance with Ethics Guidelines. This article is based on previously conducted studies and does not contain any studies with human participants or animals performed by any of the authors.

Data Availability. Data sharing is not applicable to this article as no datasets were generated or analyzed during the current study.

Open Access. This article is distributed under the terms of the Creative Commons Attribution-NonCommercial 4.0 International License (http://creativecommons.org/licenses/ by-nc/4.0/), which permits any noncommercial use, distribution, and reproduction in any medium, provided you give appropriate credit to the original author(s) and the source, provide a link to the Creative Commons license, and indicate if changes were made.

\section{REFERENCES}

1. Lim SS, Vos T, Flaxman AD, et al. A comparative risk assessment of burden of disease and injury attributable to 67 risk factors and risk factor clusters in 21 regions, 1990-2010: a systematic analysis for the Global Burden of Disease Study 2010. Lancet. 2012;380:2224-60.

2. Chobanian AV, Bakris GL, Black HR, et al. Seventh report of the Joint National Committee on prevention, detection, evaluation, and treatment of high blood pressure. Hypertension. 2003;42:1206-52.

3. Whelton PK, Carey RM, Aronow WS, et al. 2017 ACC/AHA/AAPA/ABC/ACPM/AGS/APhA/ASH/ ASPC/NMA/PCNA Guideline for the Prevention, Detection, Evaluation, and Management of High Blood Pressure in Adults: executive summary: a Report of the American College of Cardiology/ American Heart Association Task Force on Clinical Practice Guidelines. Hypertension. 2018;71:1269-324.

4. Williams B, Mancia G, Spiering W, et al. 2018 ESC/ ESH guidelines for the management of arterial hypertension. Eur Heart J. 2018;39:3021-104.

5. Mancia G, Fagard R, Narkiewicz K, et al. 2013 ESH/ ESC guidelines for the management of arterial hypertension: the Task Force for the Management of Arterial Hypertension of the European Society of Hypertension (ESH) and of the European Society of Cardiology (ESC). Eur Heart J. 2013;34:2159-219.

6. Vasan RS, Beiser A, Seshadri S, et al. Residual lifetime risk for developing hypertension in middleaged women and men: the Framingham Heart Study. JAMA. 2002;287:1003-10.

7. Huang Y, Wang S, Cai X, et al. Prehypertension and incidence of cardiovascular disease: a meta-analysis. BMC Med. 2013;11:177.

8. Muntner P, Carey RM, Gidding S, et al. Potential U.S. Population Impact of the 2017 ACC/AHA High Blood Pressure Guideline. Circulation. 2018;71:109-18.

9. The Sixth Report of the joint national committee on prevention, detection, evaluation, and 
treatment of high blood pressure. Arch Intern Med. 1997; 157:2413-2446.

10. Goff DC Jr, Lloyd-Jones DM, Bennett G, et al. 2013 ACC/AHA guideline on the assessment of cardiovascular risk: a report of the American College of Cardiology/American Heart Association Task Force on Practice Guidelines. Circulation. 2014;129(25 Suppl 2):S49-73.

11. Conroy RM, Pyörälä K, Fitzgerald AP, et al. Estimation of ten-year risk of fatal cardiovascular disease in Europe: the SCORE project. Eur Heart J. 2003;24:987-1003.

12. De la Sierra A, Segura J, Banegas JR, et al. Clinical features of 8295 patients with resistant hypertension classified on the basis of ambulatory blood pressure monitoring. Hypertension. 2011;57:898-902.

13. De la Sierra A, Banegas JR, Vinyoles E, et al. Prevalence of masked hypertension in untreated and treated patients with office blood pressure below 130/80 mmHg. Circulation. 2018;137:2651-3.

14. Banegas JR, Ruilope LM, de la Sierra A, et al. Relationship between clinic and ambulatory bloodpressure measurements and mortality. N Engl J Med. 2018;378:1509-20.

15. O'Brien E, Parati G, Stergiou G, et al. on behalf of the European Society of Hypertension Working Group on Blood Pressure Monitoring. European Society of Hypertension position paper on ambulatory blood pressure monitoring. J Hypertens. 2013;31:1731-68.

16. Blood Pressure Lowering Treatment Trialists' Collaboration. Blood pressure-lowering treatment based on cardiovascular risk: a meta-analysis of individual patient data. Lancet. 2014;384:591-8.
17. Wright JT Jr, Williamson JD, Whelton PK, et al. A randomized trial of intensive versus standard blood-pressure control. N Engl J Med. 2015;373:2103-16.

18. Filipovský J, Seidlerová J, Kratochvíl Z, Karnosová P, Hronová M, Mayer O Jr. Automated compared to manual office blood pressure and to home blood pressure in hypertensive patients. Blood Press. 2016;25:228-34.

19. Cushman WC, Evans GW, Byington RP, et al. Effects of intensive blood-pressure control in type 2 diabetes mellitus. N Engl J Med. 2010;362:1575-85.

20. Benavente OR, Coffey CS, Conwit R, et al. Bloodpressure targets in patients with recent lacunar stroke: the SPS3 randomised trial. Lancet. 2013;382:57-515.

21. ALLHAT Officers and Coordinators for the ALLHAT Collaborative Research Group. Major outcomes in high-risk hypertensive patients randomized to angiotensin-converting enzyme inhibitor or calcium channel blocker vs diuretic: the Antihypertensive and Lipid-Lowering Treatment to Prevent Heart Attack Trial (ALLHAT). JAMA. 2002;288:2981-97.

22. Thomopoulos C, Parati G, Zanchetti A. Effects of blood pressure-lowering on outcome incidence in hypertension: 5. Head-to-head comparisons of various classes of antihypertensive drugs-overview and meta-analyses. J Hypertens. 2015;33:1321-41.

23. Gupta AK, Arshad S, Poulter NR. Compliance, safety, and effectiveness of fixed-dose combinations of antihypertensive agents: a meta-analysis. Hypertension. 2010;55:399-407. 